\title{
Glucosylsphingosine Induces Itch-Scratch Responses in Mice
}

\author{
Hyoung-June KIM ${ }^{1}$, Kwang Mi KIM ${ }^{1}$, Minsoo $\mathrm{NoH}^{1}$, Hye Jin Yoo ${ }^{2}$, and Chang Hoon LeE ${ }^{3, *}$ \\ ${ }^{1}$ AmorePacific Corporation, Yongin 449-729, ${ }^{2}$ National Cancer Center, Goyang 416-769, \\ ${ }^{3}$ Department of Life Science, Dongguk University, Seoul 100-715, Republic of Korea
}

(Received July 5, 2010; Revised July 13, 2010; Accepted July 15, 2010)

\begin{abstract}
Pruritus is one of major symptoms in atopic dermatis. The pathophysiological mechanism of pruritus is unclear. The search for pruritogen is important in elucidating the pathophysiological mechanism of pruritus in atopic dermatitis. Glucosylsphingosine (Gsp) is upregulated in the strateum corneum of atopic dermatitis patients. We investigated to determine whether Gsp induces itch-scratch responses (ISRs) in mice. Intradermal administration of Gsp induces ISRs. Gsp dose-dependently induced scratching response at 50$500 \mathrm{nmol} / \mathrm{site}$ range. Pretreatment with naltrexone, an opioid $\mu$ receptor antagonist, and capsaicin, a TrpV1 receptor agonist, inhibited Gsp-induced ISRs. Additionally, Gsp-induced ISRs were also suppressed by cyproheptadine, an antagonist of serotonin receptor. These findings suggest that Gsp-induced scratching might be at least partly mediated by capsaicin-sensitive primary afferents, and the opioids receptor systems might be involved in transmission of itch signaling in the central nervous system. Furthermore, our findings suggest that Gsp-induced ISRs may be attributable to the serotonin-mediated pathways and Gsp is not any more one of byproducts of abnormal skin barrier but can lead to induce pruritus, one of typical symptoms of atopic dermatitis.
\end{abstract}

Keywords: Glucosylsphingosine, Itch-scratch response, Capsaicin, Naltrexone, Cyproheptadine

\section{INTRODUCTION}

Pruritus is an unpleasant sensation that provokes a desire to scratch. Pruritus is widely recognized as a major symptom in various allergic disorders such as allergic conjunctivitis (Bielory et al., 2001), allergic rhinitis (Cook, 1996) and atopic dermatitis (Lorette and Vaillant, 1990). Atopic dermatitis results from an interaction between susceptible genes, the host's environment, pharmacological abnormalities, skin barrier defects, and immunological factors (Leung and Bieber, 2003). Although the molecular mechanisms of atopic dermatitis remain largely unexplained, certainly an itch-scratch vicious cycle, in which scratch irritation enhances itch, is at work (Yosipovitch et al., 2003). Dryness and impaired skin-barrier functioning of the skin are clinical signs of this condition. Recently, new data from skin barrier defects has emphasized the roles of the skin barrier in atopic dermatitis. Filaggrin is a key protein involved in skin barrier functioning; indeed, mutations

${ }^{*}$ Corresponding author

Tel: +82-2-2260-8905 Fax: +82-2-2260-8769

E-mail: uatheone@gmail.com in the filaggrin gene have been shown to be important predisposing factors for atopic dermatitis (Palmer et al., 2006). Disturbed skin barrier function in atopic dermatitis is at least partly related to a disturbed lipid composition of the stratum corneum. Those symptoms are functions partly of changes in the metabolism of ceramide, which is synthesized from sphingomyelin by sphingomyelinase in the epidermal stratum corneum. In the stratum corneum of atopic dermatitis patients, however, the amount of ceramide is markedly reduced. Correspondingly, sphingomyelin hydrolysis, also in the stratum corneum, is due mainly to sphingomyelin deacylase, which converts sphingomyelin not into ceramide but instead, into sphingosylphosphorylcholine (SPC) and free fatty acid (Imokawa, 2009). The activity of sphingomyelin deacylase, in fact, is markedly higher in patients with atopic dermatitis than in healthy individuals. Glucosylsphingosine (Gsp) is increased in skin of patients with atopic dermatitis (Okamoto et al., 2003). Gsp, a metabolic precursor of glucocerebroside synthesis, also accumulates in the cerebral and cerebellar cortex in patients both with type 2 and 3 Gaucher disease (Nilsson and Svennerholm, 1982; Orvisky et al., 2002). Elevated levels of glucosylsphingosine were also seen in brains of 
type 2 Gaucher mice (Orvisky et al., 2002).

High levels of Gsp is observed in the skin of patients suffering from atopic dermatitis, but the role of Gsp in pathophysiology of atopic dermatitis is not known at all. Our continuing efforts of finding pruritogens showed that 12(S)HPETE and sphingosylphosphorylcholine (SPC) induced itch-scratch response in mice. Recently it is reported that SPC downregulates the expression of filaggrin (Choi et al., 2010). Therefore, SPC or Gsp is not any more simple byproducts of abnormal skin barrier but they can act as main factors affecting symptoms of atopic dermatitis such as itching and inflammation. Therefore we speculate whether Gsp, one of byproducts from abnormal skin barrier of patients with atopic dermatitis, induced itch symptom in mice. At first step, in this study, we examined whether Gsp induces ISRs in mice. Furthermore, in order to assess the precise mechanisms governing the Gsp-induced ISR, the effects of ketotifen, a histamine $\mathrm{H} 1$ receptor antagonist, capsaicin, a TRPV1 agonist, naltrexone, a $\mu$-opioid receptor antagonist, and cyprohepatadine, an antagonist of serotonin receptor, are described.

\section{MATERIALS AND METHODS}

All of the experiments were conducted in accordance with the Guiding Principles for the Care and Use of Laboratory Animals approved by The Korean Pharmacological Society.

\section{Animals}

Male ICR mice weighing 26-36 g were used. They were housed under controlled temperature $\left(23-25^{\circ} \mathrm{C}\right)$ and light (lights on from 08:00 to 20:00). Food and water were freely available. Each group of mice consisted of 5-8 animals.

\section{Materials}

Glucosylsphingosine was obtained from Avanti Polar Lipids (Alabaster, Ala., USA). Additional reagents included the following: ketotifen fumarate (Aldrich, St. Louis, Mo., USA) (Inagaki et al., 2000), cyproheptadine (Aldrich, St. Louis, Mo., USA) (Suh et al., 1999), and naltrexone (Sigma, St. Louis, USA) (Bienkowski et al., 1999). Additional reagents included the following: histamine dihydrochloride (Wako Pure Chemical, Osaka, Japan) (Fu et al., 1997), and capsaicin (Wako Pure Chemical, Osaka, Japan) (Holzer, 1988).

Gsp was dissolved in physiological saline, and capsaicin was dissolved in physiological saline containing $10 \%$ ethanol and $10 \%$ Tween 80 . The remaining reagents were prepared in physiological saline.

\section{Behavioral observation}

Male ICR mice 8-9 weeks of age were used in the experiments (Charles River Laboratories, MA, USA). They were housed under controlled temperature $\left(20-26^{\circ} \mathrm{C}\right)$ and light (lights on from 08:00 to 20:00), with food and water freely available. Before the experiments, the mice, the hair on the rostral part of their backs having been clipped, were put into an acrylic cage $(140 \times 32 \times 12 \mathrm{~cm})$ comprising 20 cells for at least $1 \mathrm{~h}$ acclimation. Gsp or another pruritogenic agent $(50 \mu \mathrm{l})$ was administered intradermally into the rostral region of the back. Immediately after intradermal injection, each mice was put back into the same cell and videotaped with no one present. Scratching responses to the injected site with the hind paws were enumerated as an itch-response index (Kim et al., 2008b). The number of ISRs was monitored utilizing a video camera (Sony, Tokyo, Japan) equipped with a zoom lens according to the method of Kim et al. (Kim et al., 2008b), i.e., the scratching of the rostral region of the back with either hind limb was counted.

\section{Gsp-induced ISRs}

Gsp (50 or $500 \mathrm{nmol} / \mathrm{site}$ ) was administered intradermally, and the ISRs were monitored as described above. The number of ISRs was counted every $10 \mathrm{~min}$ for 30 min following agent administration.

\section{Statistics}

All data are presented as means and S.D. Statistical significance was analyzed using two sample $t$ test and the two-way ANOVA test; $p<0.05$ was considered significant.

\section{RESULTS}

\section{Time-course of scratching behavior by Gsp}

To determine whether Gsp induces itch scratching response, Gsp was injected intradermally, and histamine, which is reported to induce the scratching response in mice, was used as a positive control. Intradermal administration of Gsp (500 nmol/site) elicited scratching of the rostral region of the back with the hind limb (Fig. 1); Scratching was first observed within 1 min post-injection in all of the mice examined, and then appeared intermittently. The scratching behavior diminished substantially after 30 min.

\section{Dose-response curve of Gsp}

Fig. 2 showed the dose-response relationship in Gsp-induced scratching. $500 \mathrm{nmol} / \mathrm{site}$ of Gsp increased the ISR number (72.66 \pm 18.14$)$ for 30 min following agent admini- 


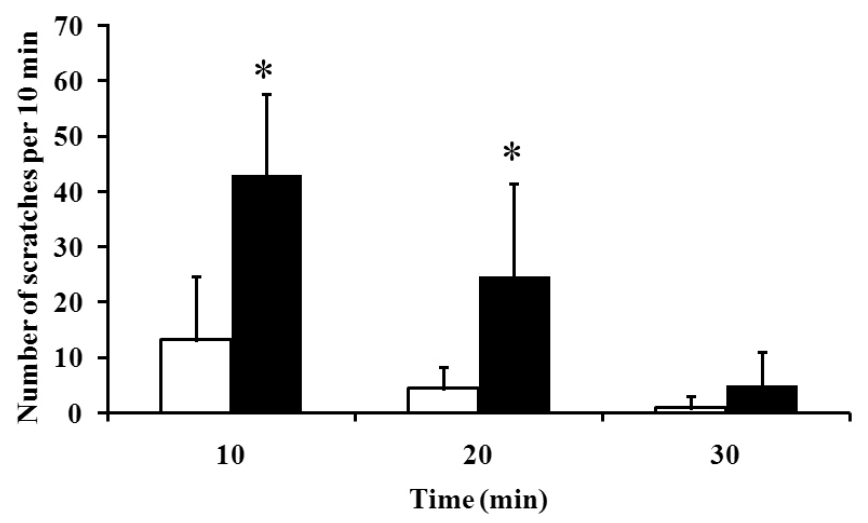

Fig. 1. Time-course graph for Gsp-induced scratching in mice. Gsp (500 nmol/site) was administered intradermally into the rostral region of the back. The number of ISRs was then observed and recorded every $10 \mathrm{~min}$ for $30 \mathrm{~min}$ following the injection. Each control group was injected with saline. The values shown represent the means and S.D. $(n=8-10){ }^{*} A$ significant difference from the control group $(p<0.05)$.

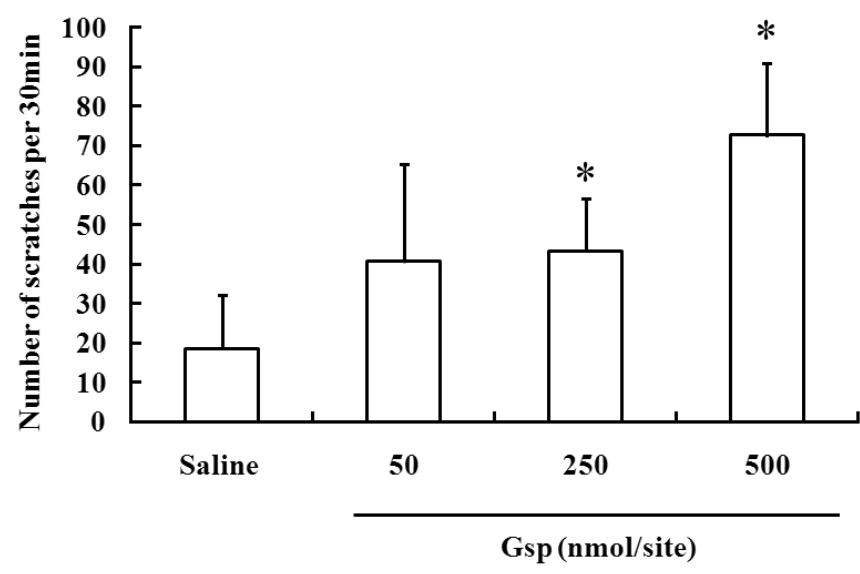

Fig. 2. Comparison of ISRs induced by Gsp (50, 150, 500 $\mathrm{nmol} / \mathrm{site}$ ) in mice. Gsp (A) or histamine (B) was administered intradermally into the rostral region of the back. The number of ISRs was observed and recorded for $30 \mathrm{~min}$. The values shown represent the means and S.D. $(n=8-10){ }^{*} A$ significant difference from the saline-treated group $(p<0.05)$.

stration. intradermal injection of vehicle solution (saline) at a volume of $50 \mu \mathrm{l}$ did not significantly elicit scratching when compared with the reactions in untreated mice (data not shown). No changes in gross behaviors other than scratching were observed after these doses of Gsp.

\section{Effect of capsaicin and naltrexone on Gsp-induced scratching}

In order to evaluate the characteristics of Gsp-induced scratching in the transmission of itch signaling, we tested whether capsaicin or naltrexon inhibited scratching in-

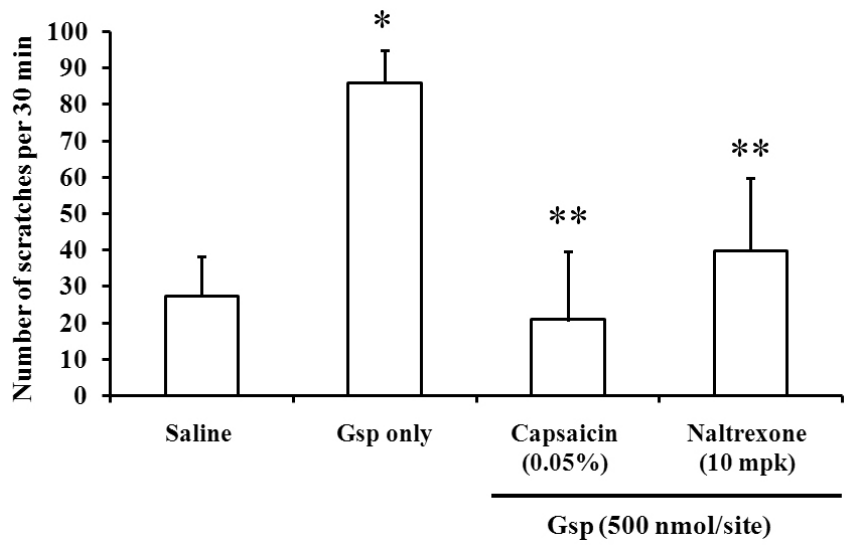

Fig. 3. Suppressive effects of capsaicin and naltrexone on Gspinduced scratching. Gsp (500 nmol/site) was injected intradermally alone (Gsp) or together with capsaicin at $0.05 \%$ (topically applied), and naltrexone (10 mg/kg, orally $1 \mathrm{~h}$ before injection), respectively. Values are the means and S.D. of eight animals. ${ }^{*} p<0.05$ when compared with Gsp control.

duced by Gsp. Capsaicin, a well-known Trpv1 receptor agonist (0.005 and 0.05\%) (Bevan and Szolcsanyi, 1990) was applied topically after the injection of Gsp, and naltrexone $(10 \mathrm{mg} / \mathrm{kg})$ was administered orally $1 \mathrm{~h}$ prior according to reports of Kim (Kim et al., 2008a). Capsaicin or naltrexone significantly suppressed the Gsp-induced scratching response in mice (Fig. 3).

Ketotifen (a histamine $\mathrm{H}_{1}$ receptor antagonist) or cyproheptadine (a serotonin receptor antagonist) is used to check the involvement of histamine $\mathrm{H}_{1}$ receptor or serotonin receptor in the transmission of itch signaling induced by Gsp. Ketotifen $(10 \mathrm{mg} / \mathrm{kg}$ ) was applied orally $1 \mathrm{hr}$ before the injection of $\mathrm{Gsp}$, and cyproheptadine $(10 \mathrm{mg} / \mathrm{kg})$ was injected intravenously 5 minute prior according to reports of Kim (Kim et al., 2008c).

The suppression by ketotifen is not significant in the Gsp-induced scratching response, however, cyproheptadine blocked the Gsp-induced scratching response in mice (Fig. 4). The Gsp-induced number of ISRs was $70.66 \pm$ 6.55; moreover, this value differed significantly from that of the saline-treated group $(p<0.01)$. The Gsp-induced number of ISRs in the cyproheptadine-treated group was 21.00 \pm 1.60 . Moreover, the value differed significantly from that of the Gsp-treated group $(p<0.05)$. No statistically significant differences were observed with respect to the vehicle-treated group.

\section{DISCUSSION}

Pruritus is a main symptom of atopic dermatitis and the mechanism of pruritus is not clear. Pruritogen is the en- 


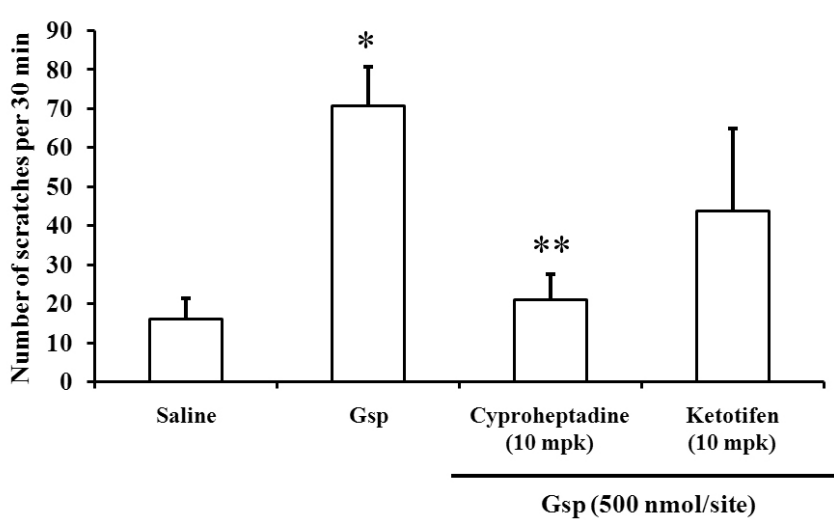

Fig. 4. Suppressive effects of the ketotifen and cyproheptadine on Gsp-induced scratching. Gsp (500 nmol/site) was injected intradermally alone (Gsp) or together with ketotifen $(10 \mathrm{mg} / \mathrm{kg}$, orally $1 \mathrm{~h}$ before injection) and cyproheptadine $(1 \mathrm{mg} / \mathrm{kg}$, intravenously $5 \mathrm{~min}$ before injection). Values are the means and S.D. for eight animals. ${ }^{*} p<0.05$ when compared with Gsp control.

dogenous compounds in inducing the pruritus or itch. The identification of pruritogen is one of ways to clarify the mechanism of pruritus and clues for therapeutics. Gsp is upregulated in the lesional skin of patients from atopic dermatitis (Okamoto et al., 2003). We speculate that abnormal skin barrier itself contributed to the symptoms of atopic dermatitis. Therefore we examined whether Gsp, one of byproducts of abnormal disruption of skin barrier in atopic dermatitis induces the pruritus, one of major symptoms in atopic dermatitis.

The results of the present study indicate that Gsp causes ISRs in mice. We found that $500 \mathrm{nmol} / \mathrm{site}$ of Gsp induced ISRs. Moreover, the maximum effect was observed during the initial 10-min period; this effect nearly subsided by 30 min following Gsp administration. Consequently, the number of ISRs was monitored for $30 \mathrm{~min}$. The results of our study indicated that Gsp induced scratching at 50 to $500 \mathrm{nmol} / \mathrm{site}$ in mice, showing a peak at $500 \mathrm{nmol} / \mathrm{site}$ (Fig. 2). It was found that, at the same concentration, Gsp elicits scratching to the less extent as histamine, LPA or SPC (Inagaki et al., 2000; Hashimoto et al., 2004; Kim et al., 2008c). Incidentally, the reason that high amounts of Gsp are required to provoke the scratch response in ICR mice might be the weak binding affinity of the putative Gsp receptor in mice or due to nonspecific binding to endogenous proteins such as albumin in mice (Han et al., 2007).

Several compounds such as capsaicin, naltrexone, cyproheptadine, and ketotifen were used to characterize the Gsp-induced ISR. Capsaicin was shown to exert inhibitory effects on the Gsp-induced ISRs (Fig. 3). These results suggested that ISR by Gsp might be at least partly mediated by capsaicin-sensitive primary afferents. Capsaicin is reported to induce the release of substance $P$ through TRPV1 from $C$ nociceptors, which desensitizes nerve fibers.

The oral opioid receptor antagonist naltrexone was also shown to exert suppressive effects on the Gsp-induced ISRs (Fig. 3). These results suggested that opioid receptor is involved in Gsp-induced USRs. Opioids have been reported to play an interesting role in itch induced not only by histamine release from dermal mast cells but also by a direct central and peripheral pruritogenic effect in addition to their major well-known role in pain (Stander and Steinhoff, 2002).

The Gsp-induced ISRs displayed a marked inhibition upon treatment with $1 \mathrm{mg} / \mathrm{kg}$ of cyproheptadine, an antagonist of serotonin receptor (Fig. 4). This finding was indicative of the involvement of the serotonin-mediated pathway in Gsp-induced ISRs. Serotonin has been reported to be an important pruritogen in mice, because dermal mast cells in rodents contain little histamine but high concentrations of serotonin (Purcell et al., 1989). It is also reported that serotonin receptors such as $5 \mathrm{HT} 1$ and $5-\mathrm{HT} 2$ receptors exist in the peripheral nerve endings (Kim et al., 2008a). Therefore, 5-HT from dermal mast cells and peripheral nerve endings might play an essential role in inducing ISR via serotonin receptors. It is not clear what mechanism is involved in the Gsp-induced ISRs. One possibility is from that Gsp mobilized the calcium via a mechanism independent of ryanodine receptor (Lloyd-Evans et al., 2003). Therefore calcium mobilization by Gsp might induce the scratch response. In fact, it was reported that phospholipase $C \beta 3$ which is a key player of intracellular calcium release, mediates the scratching response activated by histamine receptor (Han et al., 2006). Another possibility is that Gsp might induce the scratch response through G protein-coupled receptor (GPCR). Actually it is reported that lysolipid such as sphingosine 1-phosphate, lysophosphatidylic acid act via GPCR (Meyer zu Heringdorf and Jakobs, 2007). It is not identified GPCR for Gsp.

To summarize, we concluded that Gsp induces ISRs; furthermore, our findings suggest that one of byproducts from abnormal skin barrier disruption such as Gsp can lead to evoke the symptom of atopic dermatitis, i.e. pruritus.

\section{ACKNOWLEDGMENTS}

This work was supported by a Research Program for New Drug Target Discovery grant (2009-0083364) from 
the Ministry of Education, Science \& Technology, Korea.

\section{REFERENCES}

Bevan, S. and Szolcsanyi, J. (1990). Sensory neuron-specific actions of capsaicin: mechanisms and applications. Trends Pharmacol. Sci. 11, 330-333.

Bielory, L., Goodman, P. E. and Fisher, E. M. (2001). Allergic ocular disease. A review of pathophysiology and clinical presentations. Clin. Rev. Allergy Immunol. 20, 183-200.

Bienkowski, P., Kostowski, W. and Koros, E. (1999). Ethanolreinforced behaviour in the rat: effects of naltrexone. Eur. $J$. Pharmacol. 374, 321-327.

Choi, H., Kim, S., Kim, H. J., Kim, K. M., Lee, C. H., Shin, J. H. and Noh, M. (2010). Sphingosylphosphorylcholine downregulates filaggrin gene transcription through NOX5- based NADPH oxidase and cyclooxygenase-2 in human keratinocytes. Biochem. Pharmacol. 80, 95-103.

Cook, P. R. (1996). Seasonal allergic rhinitis. Mo. Med. 93, 247-250.

Fu, L. W., Pan, H. L. and Longhurst, J. C. (1997). Endogenous histamine stimulates ischemically sensitive abdominal visceral afferents through H1 receptors. Am. J. Physiol. 273, H27262737.

Han, M., Kim, Y. L., Sacket, S. J., Kim, K., Kim, H. L., Jo, J. Y., $\mathrm{Ha}$, N. C. and Im, D. S. (2007). Effect of direct albumin binding to sphingosylphosphorylcholine in Jurkat $\mathrm{T}$ cells. Prostaglandins Other Lipid Mediat. 84, 174-183.

Han, S. K., Mancino, V. and Simon, M. I. (2006). Phospholipase Cbeta 3 mediates the scratching response activated by the histamine $\mathrm{H} 1$ receptor on $\mathrm{C}$-fiber nociceptive neurons. Neuron 52, 691-703.

Hashimoto, T., Ohata, H. and Momose, K. (2004). Itch-scratch responses induced by lysophosphatidic acid in mice. Pharmacology 72, 51-56.

Holzer, P. (1988). Local effector functions of capsaicin-sensitive sensory nerve endings: involvement of tachykinins, calcitonin gene-related peptide and other neuropeptides. Neuroscience 24, 739-768.

Imokawa, G. (2009). A possible mechanism underlying the ceramide deficiency in atopic dermatitis: expression of a deacylase enzyme that cleaves the $\mathrm{N}$-acyl linkage of sphingomyelin and glucosylceramide. J. Dermatol. Sci. 55, 1-9.

Inagaki, N., Nakamura, N., Nagao, M., Kawasaki, H. and Nagai, $\mathrm{H}$. (2000). Inhibition of passive cutaneous anaphylaxisassociated scratching behavior by mu-opioid receptor antagonists in ICR mice. Int. Arch. Allergy Immunol. 123, 365368.

Kim, D. K., Kim, H. J., Kim, H., Koh, J. Y., Kim, K. M., Noh, M. S., Kim, J. J. and Lee, C. H. (2008a). Involvement of serotonin receptors 5-HT1 and 5-HT2 in 12(S)-HPETE-induced scratching in mice. Eur. J. Pharmacol. 579, 390-394.

Kim, H. J., Kim, D. K., Kim, H., Koh, J. Y., Kim, K. M., Noh, M. S., Lee, S., Kim, S., Park, S. H., Kim, J. J., Kim, S. Y. and Lee, C. H. (2008b). Involvement of the BLT2 receptor in the itch-associated scratching induced by 12 -(S)-lipoxygenase products in ICR mice. Br. J. Pharmacol. 154, 1073-1078.

Kim, H. J., Kim, H., Han, E. S., Park, S. M., Koh, J. Y., Kim, K. M., Noh, M. S., Kim, J. J. and Lee, C. H. (2008c). Characterizations of sphingosylphosphorylcholine-induced scratching responses in ICR mice using naltrexon, capsaicin, ketotifen and Y-27632. Eur. J. Pharmacol. 583, 92-96.

Leung, D. Y. and Bieber, T. (2003). Atopic dermatitis. Lancet 361, 151-160.

Lloyd-Evans, E., Pelled, D., Riebeling, C., Bodennec, J., deMorgan, A., Waller, H., Schiffmann, R. and Futerman, A. H. (2003). Glucosylceramide and glucosylsphingosine modulate calcium mobilization from brain microsomes via different mechanisms. J. Biol. Chem. 278, 23594-23599.

Lorette, G. and Vaillant, L. (1990). Pruritus. Current concepts in pathogenesis and treatment. Drugs 39, 218-223.

Meyer zu Heringdorf, D. and Jakobs, K. H. (2007). Lysophospholipid receptors: signalling, pharmacology and regulation by lysophospholipid metabolism. Biochim. Biophys. Acta. 1768, 923-940.

Nilsson, O. and Svennerholm, L. (1982). Accumulation of glucosylceramide and glucosylsphingosine (psychosine) in cerebrum and cerebellum in infantile and juvenile Gaucher disease. J. Neurochem. 39, 709-718.

Okamoto, R., Arikawa, J., Ishibashi, M., Kawashima, M., Takagi, Y. and Imokawa, G. (2003). Sphingosylphosphorylcholine is upregulated in the stratum corneum of patients with atopic dermatitis. J. Lipid Res. 44, 93-102.

Orvisky, E., Park, J. K., LaMarca, M. E., Ginns, E. I., Martin, B. M., Tayebi, N. and Sidransky, E. (2002). Glucosylsphingosine accumulation in tissues from patients with Gaucher disease: correlation with phenotype and genotype. Mol. Genet. Metab. 76, 262-270.

Palmer, C. N., Irvine, A. D., Terron-Kwiatkowski, A., Zhao, Y., Liao, H., Lee, S. P., Goudie, D. R., Sandilands, A., Campbell, L. E., Smith, F. J., O'Regan, G. M., Watson, R. M., Cecil, J. E., Bale, S. J., Compton, J. G., DiGiovanna, J. J., Fleckman, P., Lewis-Jones, S., Arseculeratne, G., Sergeant, A., Munro, C. S., El Houate, B., McElreavey, K., Halkjaer, L. B., Bisgaard, H., Mukhopadhyay, S. and McLean, W. H. (2006). Common loss-of-function variants of the epidermal barrier protein filaggrin are a major predisposing factor for atopic dermatitis. Nat. Genet. 38, 441-446.

Purcell, W. M., Cohen, D. L. and Hanahoe, T. H. (1989). Comparison of histamine and 5-hydroxytryptamine content and secretion in rat mast cells isolated from different anatomical locations. Int. Arch. Allergy Appl. Immunol. 90, 382-386.

Stander, S. and Steinhoff, M. (2002). Pathophysiology of pruritus in atopic dermatitis: an overview. Exp. Dermatol. 11, 12-24.

Suh, H. W., Chung, K. M., Kim, Y. H., Huh, S. O. and Song, D. K. (1999). Effects of histamine receptor antagonists injected intrathecally on antinociception induced by opioids administered intracerebroventricularly in the mouse. Neuropeptides 33, 121-129.

Yosipovitch, G., Greaves, M. W. and Schmelz, M. (2003). Itch. Lancet 361, 690-694. 